\title{
Recursion in language, theory-of-mind inference and arithmetic: aphasia and Alzheimer's disease
}

\author{
Zoltán Bánréti, Éva Mészáros, Ildikó Hoffmann, Zita Örley \\ Research Institute for Linguistics, Budapest, Hungary \\ https://doi.org/10.36505/ExLing-2011/04/0005/000174
}

\begin{abstract}
We found a variety of dissociations among the linguistic and non-linguistic operations tested. In the case of Hungarian speaking patients with a medium-serious $\mathrm{AD}$, syntactic recursion is relatively unimpaired, as opposed to their limited ability to tackle theory-of-mind and arithmetical recursion. Conversely, we found limited syntactic recursion but normal theory-of-mind inferences and recursive arithmetical operations in Hungarian speaking agrammatic aphasics. We argue for a model that posits a module of recursive operations in the human mind that is shared by linguistic, theory-of-mind, and arithmetical performance.
\end{abstract}

Key words: recursion, mind, aphasia, Alzheimer disease

\section{Introduction}

Hauser, Chomsky \& Fitch 2002 claim that the human faculty of recursion is revealed by syntactic-structural embeddings. Siegal and Varley (2006) argue, on the basis of experiments involving aphasic speakers, that theory of mind abilities may remain unimpaired even in cases of limited language faculties, that is, they are not grammar-dependent. Theory of mind' type reasoning can be recursive (cf. Takano -Arita 2010).

\section{Aphasics}

Bánréti (2010) conducted tests involving Hungarian speaking aphasic speakers. The results showed that, while in agrammatic (Broca's) aphasia structural recursion is limited, theory-of-mind recursion may be selectively retained. In the test situation, photographs representing everyday situations (Stark, 1998) were presented to subjects ('What is it that $X$ in the picture might be thinking of?'; 'What do you think $Y$ in the picture might be asking $Z$ to do? '). Broca's and Wernicke's aphasics gave answers whose grammatical versions were one of two types: (1) sentences involving structural recursion prompted by the structure of the question (' $X$ is thinking that he would be late'), (2) so-called situative sentences (e.g., the aphasic person impersonates $X$ and cites her thought or state of mind as in (Christ, I'll be late!') Situative sentences are non-descriptive; rather, they represent the "cited" person's thought or intention directly, in first person singular. The subjects take over the point of view of one of the characters in the picture, identify themselves with that character. It is an important fact that in such answers it is not necessary to use a syntactic structure involving clausal embedding; a simple or even fragmented clause that expresses the theory-of-mind inference is just ExLing 2011: Proceedings of 4th Tutorial and Research Workshop on Experimental Linguistics, 25-27 May, Paris, France 
enough. $73.4 \%$ of Broca's aphasics' answers were of this kind. Consequently, they were able to avoid using syntactic-structural recursion, an operation with respect to which their abilities were demonstrably limited. The use of simple situative sentences could also be observed in the case of control subjects, but only in $41 \%$ of their responses. Therefore, structural and theory-of-mind recursion represent two alternative strategies of which members of the control group were able to choose at will, whereas the aphasics were forced to choose the use of situative sentences.

\section{Subjects with Alzheiemer disease}

The validity of the above observation can be supported by cases exhibiting the converse dissociation. The same tests were conducted with the participation of the Hungarian speaking subjects with Alzheiemr disease (AD). The subjects were classified on the basis of Mini-mental state examination (Folstein, Folstein, \& McHugh, 1975, ADAS-Cog (Rosen et al, 1984). We administered the above pictures and questions ('What might $X$ in the picture be thinking of?'; 'What might $Y$ in the picture be asking $Z$ to $d o$ ?') to four persons with slight and two with medium-serious AD.

In the responses of subjects with slight $\mathrm{AD}$, there was no significant difference between them and normal controls in the proportions of replies involving syntactic-structural recursion vs. situative responses.

In the case of medium-serious $\mathrm{AD}$ the ratio of situative statements was significantly lower than in normal control subjects. Medium-serious AD subjects tend to avoid utterances in first person singular that assume the state of mind of another person. The share of sentences involving syntacticstructural recursion (embeddings involving 'that' clauses) was not lower than in normal control responses. However, $37 \%$ of these responses were semantically irrelevant statements referring to some marginal aspects of the picture. That is, while syntactic-structural recursion may remain unaffected in medium-serious $\mathrm{AD}$, theory-of-mind inferences seem to be impaired to some extent. In order to support this with additional evidence, we administered false belief tests to our two medium-serious AD subjects (following Youmans \& Bourgeois 2010). The secondary false belief test prove to be difficult for the medium-serious $\mathrm{AD}$ subjects they gave the wrong answers. Results of the secondary false belief test support the limitation of theory-of-mind inference abilities for both medium-serious AD patients.

In sum: in the slight and medium-serious stages of Alzheimer's disease, recursive clausal embedding abilities remain unaffected, but recursive theory-of-mind inferences become limited.. Unlimited syntactic-structural recursion and limited theory-of-mind inferences in Alzheimer's disease as opposed to limited syntactic-structural recursion and unimpaired theory-of- 
mind inferences in Broca's aphasia: this is a pattern of double dissociation. This finding supports theories (e.g., Zimmerer-Varley 2010) that argue for the mutual independence of these two types of recursion in adults.

\section{Arithmetic}

With respect to recursion in arithmetical calculations, we found another case of double dissociation. Following Varley et al. (2005), we gave seven different tasks to agrammatic aphasics and persons with medium-serious AD. With the latter, we found limitation in the recursion of arithmetical operations. This limitation did not concern the four fundamental operations with one-digit numbers. Our AD subjects did have an idea of numbers, and they were able to transpose numbers from verbal to visual representation, but the idea of the infinity of numbers and any operations based on it were not accessible. They were unable to produce a recursive structure (e.g., generating inserting parentheses into calculation tasks and figuring out the result) and the idea of infinity of numbers was not accessible.

The four Hungarian speaking agrammatic aphasics, apart from minor memory problems, did not exhibit any limitation in arithmetical operations, they calculated correctly, they were able to produce potentially infinite sequences of numbers, applied recursive arithmetical operations correctly, inserted parentheses in various combinations, even double ones, and came up with the correct results. They did all that using numerical symbols and operation signs, in other words, they were not necessarily able to verbalise their otherwise correct operations.

\section{Conclusion}

The accessibility of recursive operations is limited in Alzheimer's disease for theory-of-mind and calculation but unlimited with respect to linguistic representations; whereas in agrammatic aphasia, linguistic representations may be disconnected from the recursion module, whereas theory-of-mind and calculation systems are able to access it (cf. Zimmerer - Varley 2010). These dissociations argue for a theoretical model that posits a module of recursive operations in the human mind that is shared by linguistic, theoryof-mind, and arithmetical performance. This common recursion module is accessible to a limited extent for the theory-of-mind and arithmetical subsystems while it is fully accessible for representations of linguistic constructions in Alzheimer's disease, whereas in agrammatic aphasia, the representations of linguistic constructions may be detached from the recursion module while theory-of-mind and arithmetical systems may access it at will. 


\section{Acknowledgements}

This research has been supported by the National Scientific Research Fund (OTKA), project: NK 72461

\section{References}

Bánréti, Z. 2010. Recursion in aphasia, Clinical Linguistics \& Phonetics, 2010, Vol. 24, No. 11 , 906-914.

Folstein, M.F., Folstein, S.E., McHugh, P.R. 1975. "Mini-mental state". A practical method for grading the cognitive state of patients for the clinician. Journal of psychiatric research, 12/3: 189-98.

Hauser, M.D., Chomsky, N., Fitch, T. W. 2002 .The Faculty of Language: What Is It, Who Has It, and How Does It Evolve? Science, 298,1569-1579.

Rosen WG, Mohs RC, Davis KL. A new rating scale for Alzheimer's disease. American Journal of Psychiatry. 1984. Nov;14111):1356-64

Siegal, M. Varley, R., Want, S.C. 2006. Mind Over Grammar. Reasoning in Aphasia and Developmental Contexts . In Antonietti, A., Liverta-Sempio, O., Marchettio, A. (eds): Theory of mind and language in developmental contexts, Springer, 107119

Takano, M., Arita, T. 2010. Asymmetry between Even and Odd Levels of Recursion in a Theory of Mind, in: Proceedings of ALife X, ed.: L.M. Rocha, L.S. Yaeger, M.A. Bedau, D. Floreano, R.L. Goldstone, A. Vespignani www.citeulike.org/user/jasonn/article/7293338

Zimmerer, V., Varley, R. 2010. Recursion in severe agrammatism, in: van der Hulst H. (Ed.) Recursion and Human Language, 393-405. de Gruyter Mouton.

Youmans, G., Bourgeoi, M. 2010. Theory of mind in individuals with Alzheimertype dementia. Aphasiology 24:4, 515-534. 